\title{
Mathematical tales of technology and collaboration
}

\author{
Bill Blyth ${ }^{1}$
}

(Received 7 November 2010; revised 7 November 2010)

\begin{abstract}
Several tales are told about the teaching of tertiary level mathematics, with recurring themes of various forms of technology and collaboration. These include: the Australian Mathematical Sciences Institute (AMSI) program of collaborative eTeaching of advanced mathematics (at Honours level) to small classes at multiple remote sites via Access Grid Rooms (AGRs); AMSI seminars and workshops held via AGRs; some pedagogical issues with eTeaching in AGRs, a proposed pedagogical framework and examples of some software (pdf slide-show, annotations, Word, Power Point, Maple) within the context of both AGR and standard e-presentations; eMarking, where the tutor marks in an electronic environment; computer aided assessment (CAA), using generally available computer algebra systems (CAS) enabled CAA packages (the commercial MapleTA and the open source STACK ) and the more powerful CAS immersed CAA using Maple to mark individualised assignments, with plots and comments - a current research topic at RMIT University and Oxford University; and usage of CAA for
\end{abstract}

http://anziamj . austms.org.au/ojs/index.php/ANZIAMJ/article/view/3639 gives this article, (C) Austral. Mathematical Soc. 2010. Published November 16, 2010. ISSN 1446-8735. (Print two pages per sheet of paper.) Copies of this article must not be made otherwise available on the internet; instead link directly to this URL for this article. 
large classes will surely increase. The development of quality materials is resource intensive: since expertise is geographically distributed collaborative development via AGRs would be valuable.

\section{Contents}

1 Introduction

C805

1.1 The Access Grid . . . . . . . . . . . . . . C C805

1.2 The Australian Mathematical Science Institute . . . . . . . C805

1.3 eTeaching and computer assisted assessment . . . . . . . C807

1.4 Computer algebra system immersed computer assisted assessment . . . . . . . . . . . . . C 808

1.5 A call for further collaboration . . . . . . . . . . . C809

2 eTeaching: a pedagogical framework

C809

2.1 Supported didactic, or supported 'chalk and talk' . . . . . C C811

2.2 Interactive . . . . . . . . . . . . . . . . . . C812

2.3 Enhanced interactive . . . . . . . . . . . . . . C812

2.4 Research on eTeaching via the Access Grid . . . . . . . . C813

3 eTeaching: some software and Digital Ink

C815

4 eMarking

C818

5 Computer assisted assessment

C820

5.1 Computer algebra system immersed computer assisted assess-

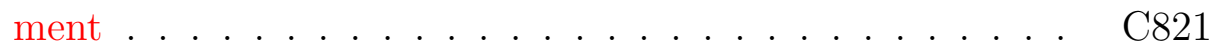

5.2 Current work . . . . . . . . . . . . . . . . . C823

6 Conclusion

C825

References

C828 


\section{Introduction}

\subsection{The Access Grid}

One of the technologies discussed here is the Access Grid which is the top end of video-conferencing systems. Video-conferencing covers a broad range of functionalities from basic two way use of video and audio for meetings to include document cameras, data exchange, desktop sharing, recording and shared applications (such as interactive whiteboards). There are many versions, mostly commercial, of video-conferencing systems available that run in specially equipped rooms or on desktops/laptops. The Access Grid (AG), developed at Argonne National Laboratories [1], has been described as videoconferencing 'on steroids': it is free, scalable to many Access Grid Rooms (AGRs), and flexible. AGRs run under Windows, UNIX or Mac environments. There is no typical AGR [1, photos]. However, AGRs usually have a 'wall': one large projection screen of three (or four) linked computer projection screens. There is usually one audio stream and video streams from three cameras. The multiple video streams from each AGR provide a good sense of 'presence', but requires high band width: so good internet connections are required.

\subsection{The Australian Mathematical Science Institute}

The Australian Mathematical Science Institute (AMSI) facilitates national collaboration in the mathematical sciences across three broad activity areas: Science; Education; and Business, Industry and Government. AMSI funded the installation of AGRs within mathematical sciences precincts of eleven of its member institutions. Additionally there are many AGRs across Australia (usually associated with high performance computing groups) so that almost all mathematical sciences departments can access a local AGR. AMSI uses AGRs to coordinate national collaboration in two major programs.

Firstly, programs of teaching of advanced mathematical sciences courses (at 
Honours level) were introduced as a pilot program with three universities in the second half of 2006. The lists of courses, with course information details, for each of the years 2007 to 2010, are published [2]. In the UK, six centres funded by the Engineering and Physical Sciences Research Council for five years, commenced (in October 2007) the teaching of broadening courses for PhD students [24]. Two of these centres use AG technology [7]. The mathematics community, especially in Australia and the UK, leads the way with collaborative teaching of advanced mathematics across networks of AGRs.

Secondly, seminars are offered via the AG. In this case, Canada led the way with it's national 'Coast to Coast' seminars: since commencement in September 2005, about 90 seminars were conducted (in the first three years of operation) via AG with various topics from mathematics, statistics and computer science. Borwein et al. [14] ellaborated a comprehensive discussion. Following the Canadian experience, AMSI coordinated AG seminars in Australia from 2007 and, in December 2007, ran a one day National Symposium on Mathematics for 21st Century Engineering Students (sponsored by the Carrick Institute for Learning and Teaching in Higher Education). This was hosted by RMIT University and 16 remote universities participated by Access Grid technology. Most presentations were given at RMIT with a local audience exceeding 55. Four presentations were made from three other AGRs (namely Monash, UQ and USQ). This was a good illustration of the collaboration that can be achieved using AG.

AMSI's AG events include seminars, distinguished lectures, AMSI workshop keynote lectures and Professor Terry Tao's Clay Mahler Lectures. AMSI publishes a list (with links to seminar abstracts) of AG events held from 2008 onwards [3]. I gave presentation protocols for lectures and seminars broadcast over the AG [9]. 


\section{3 eTeaching and computer assisted assessment}

Presentations via AG for advanced teaching and seminars raise many issues with respect to eTeaching. Section 2 proposes a pedagogical framework (which is based on a related framework for teaching in UK schools using interactive whiteboards) and comments on research on presentations in AGRs. This includes the recent research by the nGAME team at the University of Sydney that resulted in the technical development (and use) of 'history screens' in AGR Honours courses. Section 3 discusses several approaches for presentation and annotation using various software that we have experimented with, and makes some recommendations.

The advent of eLearning requires new forms of assessment. The first level of assessment still uses human markers (that is, tutors) to mark assessed work. Where this is done in an electronic environment, this has recently been called eMarking: our own work and eMarking used in the UK for public examinations (the GCSE) and at the Open University is discussed in Section 4.

The second level of assessment is usually called Computer Aided Assessment (CAA) where the marking is done automatically by computer. For several decades, many institutions have been using objective testing (that is, multiple choice questions) where, in the early years, answers were recorded on mark sense cards which were automatically marked. For example, at RMIT University, I was one of the leaders in a decade long program from the mid 1970's to conduct diagnostic testing of all students (about a thousand annually) commencing engineering and science courses. At that time, our experience was that the use of mark sense cards was unreliable so we designed our own answer sheets with transparent marking overlays which were efficient and accurate. However, the technology continued to improve: objective testing became very popular at many institutions since this was all that the technology allowed. Many users of CAA still have a 'multiple choice questions' mindset today.

However, CAA becomes a much more powerful assessment tool when students 
can input an answer without any list of the answer and possible answers ('distractors') to choose from. For example, "What is the derivative of $\sin (x)$ with respect to $x$ ?". Early attempts (such as that by Cliff Beevers [5] at Herriot-Watt University in Scotland and the CALTS project [30, 31] at RMIT University) used programmers and tricks to deal with this free user input question. The programming approach employed was to use string matching or to evaluate the student provided answer for a few $x$ values and compare to the correct values.

The most widely used Computer Algebra Systems (CAS) are Maple and Mathematica. A CAS provides symbolic calculations which are exact (as well as numeric and graphical computation). CAS-enabled CAA is the term used in the case where a CAS is used for the testing of the answer. For the example above, the CAS is used to test whether the student answer is equivalent to $\cos (x)$.

\subsection{Computer algebra system immersed computer assisted assessment}

I have been teaching using Mathematica and Maple in postgraduate and undergraduate courses since the mid 1990's. An Action Research methodology [4, 25] has been used to research and develop innovative ways of eTeaching using a CAS immersion approach. Investigations of how to take full advantage of the CAS has resulted in mathematical learning and a new, more general, class of CAA called CAS-immersion CAA. The applications and the question types of CAS-immersion CAA $[12,13]$ include marking of plots and comments and so go beyond what can be achieved with currently available CAS-enabled CAA systems. Section 5 discusses, with examples, CAS immersion teaching and CAS-immersed CAA. 


\subsection{A call for further collaboration}

I conclude with a call for further collaboration with the teaching of advanced mathematics, seminars, research; and research into eTeaching, CAS based teaching and CAA; and writing CAA assessment items. With an established network of Access Grid Rooms, project teams can draw upon members who are geographically dispersed.

\section{2 eTeaching: a pedagogical framework}

Despite the hopes and claims that Information and Communications Technology (ICT) support innovative teaching and learning in diverse discipline areas, many educational studies (usually focused on the school sector) show that there has been little change. Typically, Hughes and Ooms [17] reported from the USA that:

One major factor contributing to teachers' ill preparedness to innovatively use technologies is the lack of ongoing, focused professional learning opportunities.

The British Ofsted (Office for standards in education) reported in 2002 that:

The effect of government ICT initiatives on the quality of teaching and learning in (secondary) mathematics varies considerably among schools. Overall good practice remains uncommon.

Interactive Whiteboard (IWB) technology extends the general interactivity available with the use of data projectors. The Interactive Whiteboard is connected to the computer and a data projector in such a way that the Interactive Whiteboard behaves like a touch sensitive monitor for the computer (so that full control of the computer is effected from the Interactive Whiteboard) and freehand work is displayed (and captured, so it can be posted on the web). The closely related TabletPC's and tablet devices [20] are a more recent 
development, but now are widely used and even have international conferences dedicated to their use. Interactive Whiteboards, TabletPC's and tablet devices play a central role in the pedagogical models envisaged (and described below) with the AMSI national network of Access Grid Rooms.

British schools have been using Interactive Whiteboards for more than a decade, Dave Miller and co-workers in the Education Department at Keele University, UK, have published many research papers on the use of Interactive Whiteboards in mathematics education. From Miller and Glover, 2006 [22]:

A growing research base shows that although the use of the Interactive Whiteboard initially improves pupil motivation it need not necessarily improve teaching and learning. It is suggested that to maximise impact teachers need to move through three stages to that called 'enhanced interactive' where thinking and pedagogy change. ... However professional development is required ....

Roger Duke and Janelle Pollard from Information Technology and Electrical Engineering at the University of Queensland, in their paper on using Interactive Whiteboards in mathematics [16], also discuss phases of integrating ICT into teaching: entry, adoption, and adaptation or appropriation. The AMSI national network of AGRs (which can also join with other AGRs nationally and internationally) provides a 'quantum leap' in the level of technology available in technology-rich classrooms. However, there is a need for the development of pedagogical models of the eLearning of advanced mathematics and statistics in these technology-rich classrooms, paying special attention to the collaborative and remote learning made possible by the Access Grid.

We propose that a modification of Miller and Glover's three stages of teacher use (of interactive whiteboards for secondary mathematics education) would provide a useful starting framework for eTeaching with IWBs and TabletPCs. These three stages are supported didactic, interactive and enhanced interactive and their modified descriptions follow. 


\subsection{Supported didactic, or supported 'chalk and talk'}

The lecturer uses the basic level of the technology. The technology is often used to support slides which are prepared by a variety of methods such as PowerPoint, $\mathrm{LT}_{\mathrm{E}} \mathrm{X},{ }^{1}$ Adobe's pdf or scan to pdf and possibly several methods in combination; and Digital Ink. By Digital Ink, it is meant that handwriting and any freehand work such as diagrams are captured naturally by the software. By this means, well-known problems with the representation of advanced mathematics and mathematical diagrams and figures are completely solved. Digital Ink is provided by using a tablet device, a TabletPC or an Interactive Whiteboard. Cheap tablet devices use a stylus and the handwriting is performed on the tablet, but the output appears elsewhere on a computer monitor. Thus they are difficult to use and give a low quality result: they are not recommended. However, the tablet devices that include a monitor are easy to use and produce high quality representation of the freehand work. Interactive whiteboards act as a large touch sensitive computer monitor and the software provides for Digital Ink. The AGR provides for multiple displays (typically there are at least three projectors). The lecturer can easily, for example, have a lead presentation screen (with write-over capability), a separate screen for Digital Ink or other software (used by the lecturer or, with permission, the remote audience). Studies are needed to elucidate and compare how these technologies are best integrated into the learning environment for the lecturer, the face-to-face class and the remote class. Advice and exemplars are needed so that lecturers make an informed choice about which technologies they wish to use and how to use them effectively. In advanced mathematics (at Honours level and post graduate level) some areas of mathematics are not able to be done in a natural way using mathematical software. For examples, in topology and advanced analysis supported didactic is very likely to be the pedagogical model of choice.

${ }^{1} \mathrm{LAT} \mathrm{E}$ is the typesetting standard used in mathematics, computer science and the physical sciences 


\subsection{Interactive}

The lecturer supports the main presentation (including Digital Ink) with mathematical software such as Maple or Mathematica) or applications software such as MATLAB, statistical software, special applications software such as the commercial Finite Element Method package ANsys (which is widely used for design in many areas of engineering). The supporting software may be used to pre-prepare materials (for example, to facilitate visualization) or may be used 'live'. Students may be required to complete some work using the software. Some models of how the students, particularly remote students, are supported and engaged need to be developed.

\subsection{Enhanced interactive}

The lecturer fully integrates the use of live mathematical and applications software with presentation software and Digital Ink. The use of several screens is exploited. Students are required to become proficient with advanced mathematical and applications software. This could be described as very high technology blended learning: high quality online materials available on a Learning Management System (such as BlackBoard) with face-to-face teaching (or virtual face-to-face teaching for the remote students). This pedagogical model includes support (tutorials) for students in their use of the software. For example, the local students can easily display their file for discussion, but so too can the remote students. The remote students file can be directly controlled (remotely) by the lecturer for analysis and advice on how to proceed or on how to rectify an error.

Many variations on this pedagogical mode will be needed. It is an approach of great personal interest since we have developed a Maple immersion mode of teaching of undergraduate mathematics. (We have also used elements of this approach in an Honours Numerical Methods course and a postgraduate coursework Coding For Reliable Communications subject.) In the Maple 
immersion mode courses, the mathematical software system provides the universe of discourse: Maple is used for the presentation, word processing and typesetting, numerical computation, graphical display and symbolic computation. All teaching materials (and the examination 'paper') are electronic Maple files downloadable from the web. We have already, in August 2004, conducted a trial of this via the Access Grid with the lecturer in Brisbane in the QUT AGR and the class in the VPAC AGR in Melbourne set up in computer lab mode. This two hour session included a lecture presentation and student Maple work session where the students were assisted remotely, including help with diagnosis and correction of errors. Current work on this Maple immersion mode of eTeaching has extended to seamless integration of CAA: Subsection 5.1 gives further discussion.

\subsection{Research on eTeaching via the Access Grid}

The Access Grid was developed, initially in the USA and then also in collaboration with other countries, to support collaborative research: for a brief overview see the report on what appears to be the last of the AG Annual Retreats [6]. In Australia, the support for AG is from the Australian Research Collaboration Services, ARCS: the focus is still on research. The literature on teaching via AGRs is very sparse.

Brian Corrie and Margaret-Anne Storey [15] discuss a detailed study of the importance of gestures in distributed scientific research collaboration (via AG) arising from observations of an active research group. Over five months, 18 hours of meetings were recorded. A careful analysis of the use of gestures was completed.

An Action Research study at the host university, RMIT, of an AMSI AGR Honours mathematics course that was conducted in 2008 states [23]

This paper describes a qualitative study which was undertaken to improve the delivery methods and feedback opportunity in honours 
mathematics lectures which are delivered through Access Grid Rooms, (AGR). ... The study found that the correct placement of cameras and the use of printed material and smart boards were all crucial to the student experience. In addition, the inclusion of special tutorial type sessions were necessary to provide opportunity to students for one-on-one discussion with both lecturer and other students.

This paper includes some comments about the literature: mostly with respect to distance education. Researchers in distance education have long studied the problem of how to engage the learners that are at a distance. The Access Grid gives a greater sense of presence than other video-conference systems: this needs to be exploited to address the problem of engaging remote learners.

Recent research (soon to be published) by the national Grid for Advanced Mathematics Education, nGAme, team at the University of Sydney has resulted in the technical development (and use) of 'history screens' in AGR Honours courses. Lecturers and students were interviewed to investigate what support was desirable in teaching advanced mathematics via the AG: this uncovered a desire for 'history screens'. Some lecturers (and their students) wanted to use a supported 'chalk and talk' mode in which Digital Ink would be used for the lecture development (and another screen for asides or sketches), with the previous screen(s) displayed. It was considered to be important to mimic a standard classroom where the previous blackboard(s) or whiteboard(s) were not erased as the next part of the lecture was developed on another whiteboard. This was realised by using two tablet monitors (each with a projector for display) for Digital Ink and the use of two extra projectors to display the 'history screens' (that is, a total of five projectors; one displays video of the remote audiences). Now implemented, with attention to ease of use by the lecturer, this pedagogical development received good feedback from lecturers and students. 


\section{3 eTeaching: some software and Digital Ink}

Lecturers are increasingly using computer projection for their presentation in lectures and seminars. Mathematicians usually prefer $\mathrm{H}_{\mathrm{E}} \mathrm{X}$ for preparation of pdf notes or slides and often augment these by a separate physical whiteboard where handwriting is used for asides, worked examples and responses to questions. The beamer class in $\mathrm{HT}_{\mathrm{E} X} \mathrm{X}$ is recommended to create a pdf slide show. An attractive feature (especially if only one projection screen is being used) is the $\mathrm{H}_{\mathrm{E}} \mathrm{X}$ package multimedia which is provided to run movies, but can be used to hyperlink to any software (such as Word and Maple). After the external software has been run, an exit from that software (whether Word, Maple, ...) produces a return to the slideshow. This is very useful since the main presentation can be prepared from $\mathrm{H}_{\mathrm{E}} \mathrm{X}$, with correct mathematical typesetting: the slideshow is suspended while software demonstrations are run ... and when completed, the slideshow is resumed. I detail discussions and references for this (and other topics in this section) elsewhere [8, 10].

Normally, pdf files are used for publishing or presenting work in a final state: the pdf file is prepared with $\mathrm{AT}_{\mathrm{E}} \mathrm{X}$ and printed or viewed from Adobe Reader. In a presentation, focus on a particular element can be achieved in several ways: a laser pointer is effective in a local presentation, but not for distributed meetings via the Access Grid (since the Access Grid transmits a copy of the computer screen via VNC). Slideshows produced using beamer can provide stepped uncovering of parts of the slide and thus provide some focus.

However, the software pdf Annotator (which costs about Us\$50) or Jarnal (which is open source and free) can be used to freely add annotations to pdf documents and to do highlighting. The annotations can be text or handwriting. This can be done with a normal mouse but handwriting with a mouse is difficult, slow and very jerky: it is far superior to use a pen with a tablet device. Some cheap tablet writing pads, connected via a USB port, enable writing with a pen/stylus but the writing only appears on the computer monitor (not directly where the writing is performed). A TabletPC has a pen 
and a touch sensitive monitor, so writing (directly on the monitor) is easy and natural (as it is also for the Wacom tablet monitors). Freehand writing and sketching is called Digital Ink.

As well as annotation of a presentation as discussed above, the capability to annotate pdf files is valuable in several contexts:

1. annotate a blank pdf file for a 'chalk and talk' lecture;

2. live working of an example in the lecture;

3. direct student work, by hand, using a tablet device then print to pdf;

4. student handwritten work on paper and scanned to pdf for e-submission;

5. eMarking student work, submitted as pdf files, and returned as pdf files. We return to eMarking in the next section.

For those who choose to use Word, PowerPoint or Excel and would value Digital Ink and annotations, using a TabletPC provides a very attractive environment: an extra toolbar enables easy and natural Annotation and Highlighting. Microsoft also provides Windows Journal and Microsoft OneNote to support Digital Ink. As a cautionary tale, we mention that a recent investigation into boredom amongst UK university students [21] concluded

The most boring teaching methods were found to be laboratory work, computer sessions and copying lecture notes whilst the most significant contributor to lecture boredom in terms of teaching method is the use of PowerPoint (without a handout).

The discussion in the paper is more balanced than this quote might indicate: clearly PowerPoint is a tool and it's efficacy depends on how it is used.

Maple (and Mathematica) provide much more than numeric, symbolic and graphical computation with full programming languages. Additionally, there is word processing, providing very good mathematical representation with full palettes of symbols, and slideshow modes for presentations. Maple supports 
Digital Ink: but only in a special rectangular region called a Canvas. This has some pedagogical possibilities: for example, the live working by hand of a problem in the lecture where Maple can be used to typeset the problem and provide an appropriate plot, before the solution is developed by hand [10, e.g.]. The Maple developers plan to provide the facility to annotate freely by hand (similar to the complete freedom to annotate Microsoft packages such as Word): this will provide clear pedagogical advantages for teaching and presentations using Maple.

For some comments on the pedagogy of interactive whiteboards, see Section 2 . Note that interactive whiteboards typically:

1. have many drawing tools and an eraser,

2. can draw over any software, but annotations are not saved in that software,

3. can export to jpeg, pdf, html.

The second item is the greatest strength and greatest weakness of annotating with an IAW. All of the annotation methods described above are specific to the underlying software being used and allow the annotations to be discarded or saved to the underlying software. If your favorite software does not support annotations, then you cannot annotate. IAWs work differently: a SMART Board has a tray of four different pens and an eraser. When all pens (and eraser) are on the tray, the operation of all software and windows is controlled by the mouse. The mouse can be a finger, another pen (on the SMART board) or the mouse from the connected computer ... or a mouse device of a remote collaborator in another AGR (if remote control is enabled). In this way, software such as Maple can be used.

To annotate any part of the IAW screen, pick up one of the pens and write or draw independently of whatever software is being used! In an AGR, a remote collaborator's mouse can annotate anything displayed.

The cost of this wonderful flexibility is that the annotations are not connected 
with the underlying software, but they can be saved in the IAW's file. Annotations for emphasis would not usually be saved. However, a blank page of the IAW software could be used [10, e.g.] for Digital Ink (to work an through an example or to sketch by hand) which can be saved, edited and distributed.

\section{4 eMarking}

Student work using some computer package has existed for decades, starting from coding in some programming language such as FORTRAN. Usually computer output was appended to (or inserted in) handwritten work for submission. However, the use of CAS such as Maple (or Mathematica) provides a new environment for doing mathematics: new learning requires new forms of assessment. In 2004 Blyth and Labovic [11] discussed eAssessment implemented in a variety of forms for three courses at third year level and two courses at first year level, where all of the courses are Maple based. In summary, typically, assignments are individualized and completed in small groups. At that time, the Maple assignment files were emailed by students to proxy email accounts (to avoid standard email accounts being overrun by assignment files). Recently, a Learning Management System (namely BlackBoard) has been used and is recommended.

Our eMarking comment for one of the assignments [11] follows:

For marking, we spent about two hours discussing our new approach and pre-preparing the text comments that we anticipated would be appropriate. In the lecturer's solution file, a Marking section holds a collection of all of the comments inserted into the student files and a template marks summary in green text cells that were easily recognizable from the usual Maple black text, red input and blue output: a brief sample is given in section 4 below [11]. The assignment Maple files were opened [by the tutor] (from the email proxy account), comments, marks and feedback 
inserted; and returned by e-mail.

The marking took about six hours for 27 submissions. A rate of five per hour might seem a little slow, but it should be remembered that the assignments were individualized: the solution file was executed for each student group's submission. It is important to note that the comments, cut and pasted, for feedback were more extensive than would have been provided had the work been marked by hand.

This eMarking (that is, marking in an electronic environment by human tutors) of individualized assignments can be a little costly in terms of staff resources. This leads naturally to a consideration of CAA (see the following section). We note that the assignment mentioned above no longer exists, but with the CAS-immersion CAA approach (discussed in the next section), we estimate that preparation of the Maple files would take at least ten hours (up to even 30 hours in some cases), but the marking (including marking plots by the tutor) would take about one hour and uploading the marks to BlackBoard is only a few minutes, but uploading the Marking Reports is not yet well supported by BlackBoard and would take about another hour. Since the assignments are individualized, they can be re-used. However, the preparation time becomes well spent for large classes (and for assignments which would otherwise be slow to mark).

Section 3 mentions the use of Digital Ink for eMarking. Digital Ink is now well supported and has already been used in an AGR Honours mathematics course by Andrew Stacey and Yousong Luo of RMIT University in 2008 [8]: assignments were completed by hand, scanned to pdf, emailed, marked by the tutor with pdf Annotator and emailed back to the student. In 2009, Ben Mestle's team at the Open University in the UK used 32 tutors covering nine courses in their MSc program to develop and trial an electronic tutor-marked assignment, eTMA, system for mathematics [18]. eSubmission was optional, but about $20 \%$ of students elected to use eTMAs and these students responded positively about the eTMAs. Tutors mostly chose to use a TabletPC and 
pdf Annotator and were provided with hardware, software and professional development sessions. Alternatively, tutors could choose to use a tablet device or Word 2007. Interestingly, over $90 \%$ of students who used eSubmission also prepared their work electronically, with $\mathrm{AT}_{\mathrm{E}} \mathrm{X}$ being the most popular, followed by Word. Feedback from tutors was positive and comments indicated that a time overhead (compared to paper marking) would probably be about $10 \%$ in the long run. They concluded [18]:

Although e-marking of mathematics is still in its infancy, its use is likely to grow as universities become increasingly digitised and students come to expect and demand electronic submission of their assignments.

\section{Computer assisted assessment}

As mentioned in the Introduction, CAA has evolved from the use of multiple choice questions to CAS-enabled CAA systems, the first of which was Aim in 2000 [26, 27, 28, 29, e.g.]; Keady et al. [19] compared several CAA systems. I do not favour the use of multiple choice questions given the availability of CAS-enabled CAA, but note that [28]:

The current paradigm in CAA is still the multiple choice question (MCQ) or similar (multiple response etc.) in which teacher provided answers are selected by students.

The evaluation of student provided answers is greatly to be preferred to teacher provided answers [28]. Unfortunately Aim (which was excellent) is no longer maintained, but MapleTA (a commercial product from the Maple people) and STACK (an open source system authored by Chris Sangwin) are widely used.

The Aim system was open source, used Maple for its CAS engine and was adopted by many universities. With Aleksandra Labovic, we inspected the 
code for Aim and wrote (and tested) an Aim question on the Trapezoidal and Simpson rules for one of our first year Maple based classes [11]. However, this Aim assignment was restricted compared to the actual Maple assignment. The Maple assignment [11] asked for a log-log plot of the trapezoidal error (of a parameterized quadrature problem) and hence to show that the error followed a power law. Further modification of the code was required of the student, particularly to code Simpson's rule (given code for the trapezoidal rule). The affordances of CAS-enabled CAA are great for testing and marking exact (symbolic) answers and numerical answers, but the constraints include not being able to mark plots and comments.

\subsection{Computer algebra system immersed computer assisted assessment}

Since we teach in a Maple immersion mode in which our students are expected to become proficient users of Maple, from 2005 onwards we implemented CAS-enabled CAA features in our Maple learning files. No external package is needed for the CAA, and the usual syntax problems are not an issue. The CAA is integrated seamlessly into the learning activity: the CAA component is written (that is 'authored') in a separate Maple file as a procedure that is saved in Maple's binary form. Both of the Maple file for the activity and the binary file with the marking procedure are downloaded by the student. The student Maple activity file includes the command to open the required file so that when activity (or part thereof) has been completed, the student executes the marking procedure (with the appropriate arguments) to have a marking report displayed on the screen [12]. The first such activity was 'Spot the Curve' in which three ways of identifying the translation used to move an initial curve (a quadratic or a cubic) to its image curve. The translation was a randomly generated value of the translation of $a$ (respectively $b$ ) in the $x$ (respectively $\mathrm{y}^{-}$) direction, so the correct answer was a pair of numbers. Thus the usual CAA testing (as implemented in AiM) can be used: for marking, a conditional statement tests whether the difference between the correct value 
and the student response value is zero. However, the Spot the Curve activity, at the simplest level, requires students to manipulate plots that have been generated automatically until the student plot is identical to the provided plot. Next, students use animation of translated curves displayed with the provided (target) curve to identify the translation. This project received very positive feedback from students [12]. Note that this activity cannot be done using CAS-enabled CAA since the plots might be able to be generated by the CAA system, but they cannot be made available for use in a separate software package for direct use as required here. We suggest that this is the first of a new class of CAS-immersed CAA.

As details of the first year course, and the student groups taking it, changed to include science students who were not majoring in mathematics, we decided to greatly simplify the trapezoidal rule assignment (and Simpson's rule was moved to another course). We created the FishPond assignment which asked how much money a trout farmer would earn: the substantial part of the activity was to use the trapezoidal rule to compute the area of the pond [12]. The student Maple file read in a procedure to create a plot of the parameterized FishPond with horizontal cross sections drawn and labeled with the corresponding width, a provided a list of widths (so students did not need to copy numbers from the plot) and a full marking procedure. The student could execute the marking procedure and view the displayed marking report. Without penalty, students could repeat any part of their work: when satisfied, they saved their file and sent to the tutor who executed the file and noted the mark for entry into the marks list. Students really enjoyed this activity and we noticed that students were very focussed on trying (by re-doing parts marked as incorrect) to get full marks: their behaviour reminded us of game players trying to 'clock' a game.

In the second half of 2008, we addressed the issue of constraints. We want to assess what we want to assess, not what the tool allows; thus we want to be able to mark plots and comments. In a CAS-immersed CAA, we created Maple templates for the student to use for their work in which the answers to be marked are saved to specified names. As well as symbolic and numerical 
answers, this includes saving plots and comments and was implemented for the Pendulum problem [13]. A marking procedure was read into the student's Maple template file which allowed for all components which can be automatically marked to be marked immediately by students: the displayed marking report gave the marks 'so far'. As previously, students could recompute any part of their work without penalty. Students were experienced Maple users by now (near the end of first year) and they needed very little help in the computer labs: this allowed us to spend most of the time observing student behaviour. Even though the automatic marking was for only part of the assignment, student behaviour was much the same as for the FishPond assignment: they reminded us of game players trying to 'clock' a game. Formal student feedback was also very positive [13].

After the student submits their Maple file (knowing the marks 'so far'), the tutor executes the student file and then executes the tutor's marking file (in shared kernel mode). The remaining components of plots and comments are assigned marks by the tutor in an efficient manner by viewing the correct plot (respectively comment) alongside the student plot (respectively comment) and entering the appropriate mark. In 2008 the displayed Marking Report was copied and pasted into the student Maple file (uploaded to BlackBoard) and the mark entered into the BlackBoard eGradebook [13]. Table 1 gives a dummy full marking report for the Pendulum problem

The symbolic answers [Q1(a), Q2(a) and Q2(b)] and the numeric answer [Q2(c)] are marked automatically and immediately by the student and the tutor adds in the marking for the comment answer [Q1(b)] and plots [Q1(c) and Q2(c)]. No other CAA system provides for marking of comments and plots, either automatically or semi-automatically.

\subsection{Current work}

In addition to the first year work with Labovic, I have been developing a Maple immersion mode of teaching more advanced courses, mostly in third year. 
TABle 1: A copy of a dummy full marking report for the Pendulum problem. Marking report for student numbers:

$3078453,3087312,3077450$

Student names are: Bill Blyth, Aleksandra Labovic, Robin Hill

The group number used was: $g p N=110 / 3$

Total Marks: 8 (out of 10)

Your Q1(a) particular solution is correct; 1 mark.

Your Q1(b) ic comments ... 1 marks (out of 2 marks)

Your Q1(c) plot ... 1 marks (out of 2 marks)

Your Q2(a) general solution is correct; 1 mark.

Your Q2(b) particular damped solution is correct; 1 mark.

Your Q2(c) plot ... 2 marks (out of 3 marks)

Your Q2(d) value of $\mathrm{k}$ for maximum damping is correct; 1 mark.

The Geometry of Surfaces course includes automatic marking procedures for exercises on many of the earlier topics in the course. However, the Vector Calculus course was rewritten in 2009 to adapt the CAS-immersion CAA approach to the assessed work. An additional advantage of this approach is easy to see: Maple uses special types for Vectors and Matrices and also for VectorFields. So besides extending the CAA to include marking of plots and comments, the student's objects such as VectorFields are dealt with naturally (which would not be the case with some CAS-enabled CAA such as STACK for which a VectorField is not a valid entry type).

Also in 2009, I and Cath Wilkins wrote CAS-immersed CAA templates for the Maple Explorations first year course at Oxford. We conducted a small trial, and gave a joint presentation at the CETL-MSOR Conference in September 2009, of this unpublished work. Two of the projects were rewritten and the CAS-immersed CAA was extended to include marking of algorithms (and proofs). The templates included writing an algorithm as a procedure and 
saving to a name. This allowed testing the algorithm for validity (as well as for the results that were required) and to print the algorithm so that it could be assigned a mark for elegance. Also, the marking procedure was refined so that the Marking Report was printed to a txt or an rtf file (not sent to Oxford students since the only feedback allowed was the final mark), and a cumulative Marks List (for results processing) was auto generated.

Further improvements to the formatting of the Marking Report, the authoring process and the provision for extra optional student feedback have been implemented for the Vector Calculus course in 2010. Assignment 3 (based on a question from the 2004 examination) was parameterized and students (in small groups) needed to use line integration to evaluate the areas of walls of a shed and use double integration to evaluate the volume of the shed. Table 2 gives a slightly artificial dummy report for assignment 3 .

Note the improved format of the Marking Report (now generated automatically, without manual formatting), and each subpart of a question for which marks are given has an optional extra comment line. For example, the first subpart of Part(a) has a non-null extra comment, so it is printed with a small indent. The generation of the Marking Report tests whether the extra comment for every marked component is non-null: if no extra comment has been made, then no line(s) are printed.

\section{Conclusion}

The AMSI program of collaborative eTeaching of advanced mathematics to small classes at multiple remote sites across the country successfully uses Access Grid technology. The Access Grid Rooms are the most technology-rich classrooms in the country: a start has been made on developing a variety of pedagogical styles and a framework of such, but more work is needed (particularly on the proposed Interactive and Enhanced Interactive modes). The AMSI AGR seminar program now includes special seminars and lecture 


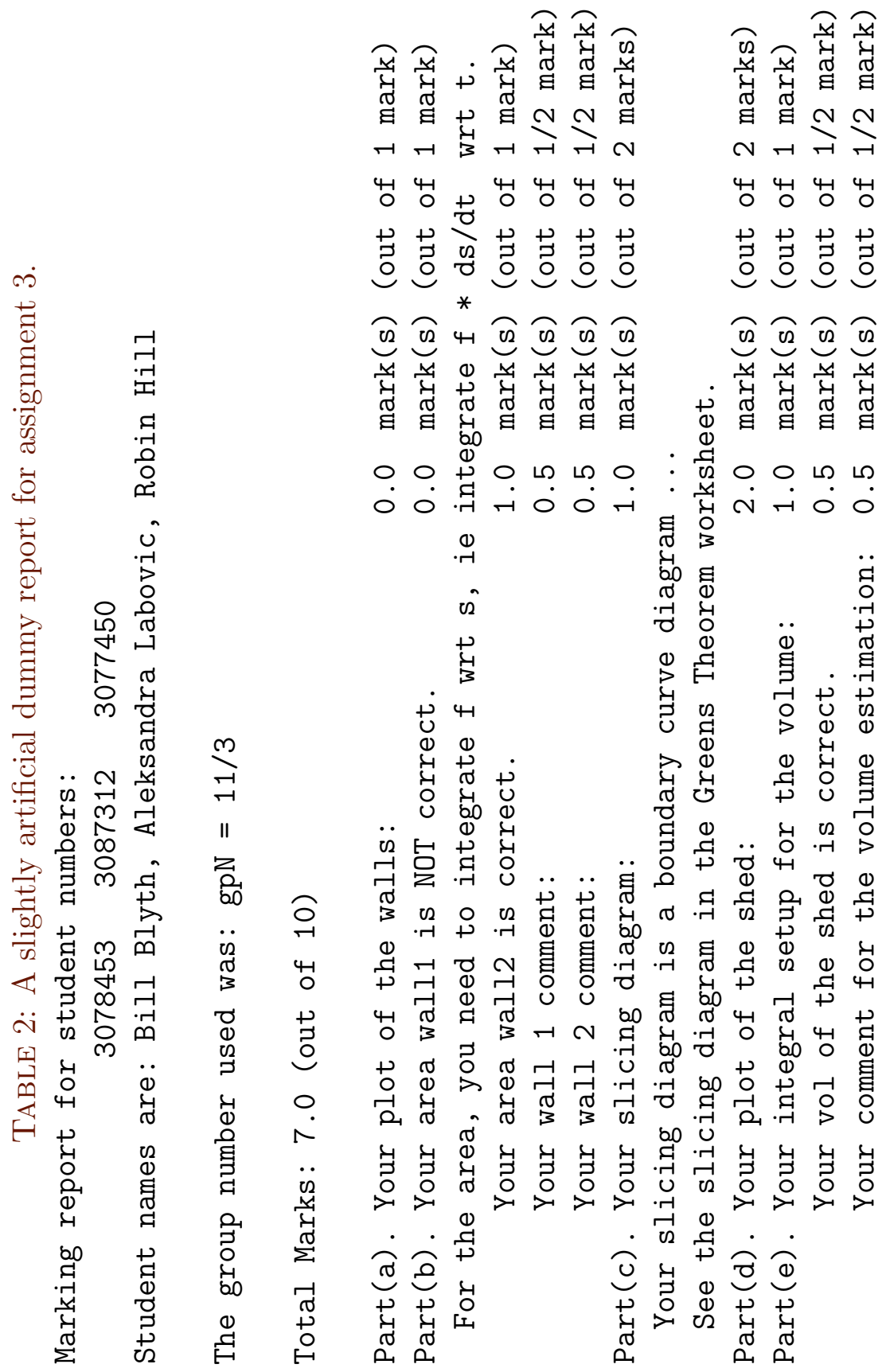


series where keynote speakers at AMSI supported conferences and workshops give presentations to national audiences via AGRs. As an example, the ClayMahler Lecturer for 2009 was Terry Tao, Fields Medalist. One of his lectures, given in the AGR at the University of Western Australia, had a national audience via 16 AGRs across the country. This included participation in the event that was held in Perth from James Cook University in Townsville: an opportunity facilitated by the technology and cooperation.

The technological support for Digital Ink enables some pedagogical styles via the AG, but also makes eMarking with hand writing by a tutor easy and efficient. The relatively low overheads (compared to hand writing on hardcopy assignments) will make this appealing for some eSubmission of work: for example, at the Open University and for some of the AMSI AGR honours mathematics courses which have small enrolments.

For completely automatic marking, CAS-enabled CAA such as provided by MapleTA or STACK are excellent: students download their problems from the system and enter their answers into the system. Marking is done automatically (using the CAS 'under the hood') and results are entered into a database and directly into the electronic grade book of the Learning Management System. This is ideal for lower level activities such as drill and practice at school level and some first and second year level university courses. Resources to develop appropriate assessment items are needed: since there are eight Australian universities using MapleTA, I propose that collaborative development for assessment items for various courses at first and second year be undertaken. With mathematical preparedness of students entering the universities an issue of concern, and the student to staff ratio continuing to worsen, the use of some CAA for large enrolment classes continues to become more and more attractive. Although different universities have different standards, content and style in a given course, there would be sufficient overlap that a collaborative project facilitated by use of the AG would be valuable.

Disadvantages of CAS-enabled CAA include, firstly, the CAA is an external package for which calculations are done elsewhere (on paper or with some 
calculator or computer package) and then entered into the CAA; and secondly, the question types are relatively limited to only those with symbolic or numeric answers. I have been working on a Maple immersion mode of eTeaching with an objective of taking full advantage of the CAS, Maple, with new pedagogy and curriculum. Inevitably, new forms of assessment are also needed which had lead to CAS-immersed CAA in which plots, comments, proofs and algorithms are also marked. Our aim is to maximise affordances (of the CAA) to 'everything' and to mimimise the constraints to 'essentially none' ... provided that the students are proficient users of the CAS. Considerable development of the CAS-immersion CAA approach has been achieved, bur more research needs to be done. Further work includes the creation of a Mathematica version. I invite collaboration and suggest that Access Grid technology would be ideal to facilitate collaborative research and development with a geographically dispersed project team. More generally, I suggest that research into eTeaching, CAS based teaching and CAA is needed and AG technology can facilitate this.

Acknowledgements My position at AMSI is, in part, supported by the Collaboration and Structural Reform project, nGAME, funded by the Australian Government and based at the University of Sydney.

\section{References}

[1] Access Grid. http://www.accessgrid.org. C805

[2] AMSI-Mathematical Sciences Access Grid Honours courses (and course desriptions). 2007-2010. http://www.amsi.org.au/index.php/ ice-em/higher-education/access-grid/subjects-a-courses. C806 
[3] AMSI-Mathematical Sciences Access Grid events and seminars. http://www.amsi.org.au/index.php/ice-em/higher-education/ access-grid/events-and-seminars. C806

[4] R. Baskerville. Investigating information systems with action research. Communications of the Association for Information Systems, 2(Article 19), 1999. C808

[5] C. Beevers. Computer Assisted Learning in Mathematics, publications from 1985 to 2008. Available at http://www.calm.hw.ac.uk/publications.html. C808

[6] B. Blyth. Access Grid Retreat 2008 Report. AustMS Gazette, 35, 4 (September), 254-257, 2008. http:

//www . austms . org. au/Publ/Gazette/2008/Sep08/CommsAGR. pdf. C813

[7] B. Blyth. What is Access Grid? ... and what is it good for? AustMS Gazette, 35, 5(November), 311-314, 2008. http://www . austms.org.au/Gazette. C806

[8] B. Blyth. The Access Grid: Australia - New Zealand collaboration and eTeaching with pdf. AustMS Gazette, 36, 1 (Mar), 17-21, 2009. http: //www . austms . org. au/Publ/Gazette/2009/Mar09/AccessGrid.pdf. C815, C819

[9] B. Blyth. The Access Grid: AG seminars: protocols and using VLC media player. AustMS Gazette, 36, 3 (July), 183-187, 2009. http: //www. austms.org.au/Publ/Gazette/2009/Jul09/AccessGrid.pdf C806

[10] B. Blyth. Digital ink and annotations in e-teaching of advanced maths. MSOR Connections: special edition featuring articles following the CETL-MSOR Conference 2008 (Continuing Excellence in the Teaching \& Learning of Maths, Stats \& OR), vol 9, no 4, November 2009January 2010, 18-22, 2010. Published by the Maths, Stats \& OR 
Network, UK. Available at http:

//www.mathstore.ac.uk/headocs/9418_blyth_b_digitalink.pdf C815, C817, C818

[11] B. Blyth and A. Labovic. Assessment of e-Mathematics with Maple. In W.-C. Yang et al., editors, Proc. 9th Asian Technology Conference in Mathematics: ATCM 2004, 143-152. ATCM Inc, USA. C818, C821

[12] B. Blyth and A. Labovic. Active Learning and Fun in First Year using Maple. In P. H. Kloppers, C. S. Joubert and M. E. Joubert, editors, Buffelspoort TIME2008 Peer-reviewed Conference Proceedings, Technology Integration into Mathematics Education (TIME) Conference, Buffelspoort, South Africa, September 2008. Published (CD and online) ISBN: 978-0-620-43454-6, 2009, by Tshwane University of Technology, Pretoria. http:

//time.tut.ac.za/public/(1)_Peer-Reviewed_PROC_TIME2008.pdf. C808, C821, C822

[13] B. Blyth and A. Labovic. Using Maple to implement eLearning integrated with Computer Aided Assessment. Int. J. Maths. Ed. Sci. Technol., 40(07), 975-988, 2009. doi:10.1080/00207390903226856. C808, C823

[14] J. Borwein et al.. Coast-to-Coast (C2C) Seminar: Background, History, and Practice; and Apendices A \& B. In J. Borwein, E. M. Rocha and J. F. Rodrigues, editors, Communicating Mathematicsin the Digital Era. AK Peters, 2008. Available from http://users.cs.dal.ca/ jborwein/c2c08.pdf. C806

[15] B. Corrie and M. A. Storey. Towards understanding the importance of gesture in distributed scientific collaboration. Internat. J. Knowledge Informat. Systems, 13(2), October 2007. Springer. doi:10.1007/s10115-006-0062-2. C813

[16] R. Duke and J. Pollard. Case Studies in Integrating the Interactive Whiteboard into the Secondary School Mathematics Classroom. In 
W.-C. Yang et al., editors, Proc. 9th Asian Technology Conference in Mathematics: ATCM 2004, 169-177. ATCM Inc, USA. C810

[17] J. E. Hughes and A. Ooms. Content-focussed technology inquiry groups: preparing urban teachers to integrate technology to transform student learning. J. Research on Tech. in Educ., 36.4, Summer 2004. C809

[18] C. Jordan, G. Arrowsmith, T. Lowe and B. Mestel. Electronic marking in mathematics - the marker and student perspectives. MSOR Connections, 10, 1, 43-47, 2010. Maths, Stats and OR Network, UK. C819, C820

[19] G. Keady, G. Fitz-Gerald, G. Gamble and C. Sangwin. Computer-aided assessment in mathematical sciences. In Proceedings of the Assessment in Science Teaching and Learning Symposium, The UniServe 2006 Conference, 69-73. UniServe Science 2006. Available at http://science. uniserve.edu.au/pubs/procs/2006/keady.pdf. C820

[20] B. Loch and D. Donovan. Progressive Teaching of Mathematics with Tablet Technology. Journal of Instructional Science and Technology, e-JIST, Vol. 9, No. 2, 2006. Accessed via www. usq.edu.au/electpub/e-jist/docs/vol9_no2/papers/ current_practice/loch_donovan.htm. (e-JIST recently merged with the Australasian Journal of Educational Technology, visit http://www.ascilite.org.au/ajet/ajet.html). C809

[21] S. Mann and A. Robinson. Boredom in the lecture theatre: an investigation into the contributors, moderators and outcomes of boredom amongst university students. British Educational Research Journal, 35(2), 243-258, April 2009. C816

[22] D. J. Miller and D. Glover. Evolution for a revolution: professional development for mathematics teachers using interactive whiteboard technology. In The Seventeenth ICMI Study: Technology Revisited. Hanoi. 2006. C810 
[23] L. McArthur, L. Klass, A. Eberhard and A. Stacey. Action research to improve methods of delivery and feedback in an Access Grid Room environment. Submitted. Available from

Lynne.McArthur@rmit.edu .au. C813

[24] New mathematical sciences taught courses. Connect, 49, April 2007, p.3. Available from http://www.epsrc.ac.uk/CMSWeb/Downloads/ Publications/Connect/Connect49.pdf. C806

[25] R. O'Brien. An Overview of the Methodological Approach of Action Research. 1998. Available at http://www.web.net/robrien/papers/arfinal.html. C808

[26] C. J. Sangwin. (2003). Assessing Higher Mathematical Skills Using Computer Algebra Marking Through AIM. In R. L. May and W. F. Blyth, editors, EMAC2003 Proceedings, Proceedings of the Sixth Engineering Mathematics and Applications Conference, 229-234. Engineering Mathematics Group, ANZIAM, Australia, 2003. C820

[27] C. J. Sangwin. Assessing mathematics automatically using computer algebra and the internet. Teaching Mathematics and its Applications, 23(1), 1-14, 2004. http:

//web.mat.bham.ac.uk/C. J.Sangwin/Publications/tma03.pdf. C820

[28] C. J. Sangwin and L. Naismith. Implementing Computer Algebra Enabled Questions for the Assessment and Learning of Mathematics. International Journal of Mathematical Education in Science and Technology, 15(1), 3-18, 2008. C820

[29] P. S. Siew. Flexible Online Assessment and Feedback for Teaching Linear Algebra. Int. J. Math. Sci. Technol., 34(1), 43-51, 2003. C820

[30] E. Yu and W. F. Blyth. A CAL and Testing System and student streaming. In J. A. Bowden and S. Lichtenstein, editors, Student Control of Learning: Computers in Tertiary Education, Proceedings of 
the CALITE-85 Conference, 391-396. Published by The Centre for the Study of Higher Education, University of Melbourne, 1985. C808

[31] E. Yu and W. F. Blyth. CALTS-An integrated and interactive CAL environment. K. Fielden, F. Hicks, and N. Scott, eds., In Computers in Learning in Tertiary Education, Proceedings of the ASCILITE-88 Conference, 253-264. Published by School of Information Sciences, Canberra C.A.E., 1988. C808

\section{Author address}

1. Bill Blyth, Australian Mathematical Sciences Institute, University of Melbourne, Australia.

mailto:bill@amsi.org. au 